\title{
Rehabilitación Psicosocial: Un enfoque efectivo para ayudar a personas con enfermedades mentales agudas
}

\section{Psychosocial Rehabilitation: An effective approach for helping persons with serious mental illness}

\section{RICHARD BOETTCHER}

Ph.D. Richard E. Boettcher es Profesor Emérito en la Facultad de Trabajo Social de la Universidad de Estado de Ohio. Su dirección en la Escuela de Trabajo Social es 1947 College Road Columbus, Ohio 43210; su dirección de correo electrónico es: Boettcher.1@osu.edu

\begin{abstract}
Resumen
Este artículo reconoce en las enfermedades mentales un problema de salud global con importantísimas consecuencias en el ejercicio de los derechos humanos y da cuenta de la evaluación de un programa para personas afectadas por enfermedades mentales, centrado en la rehabilitación psico-social con base comunitaria que se realizó en Columbus Ohio, Estados Unidos. El estudio utilizó un diseño experimental y encontró que los sujetos del grupo de tratamiento psicosocial superaron a los sujetos del grupo de control en las variables claves de resultados de cantidad de re-hospitalizaciones, cantidad de días de hospitalización, visitas de urgencia a los servicios de salud mental, y contactos con el sistema de justicia penal. Los sujetos del grupo de tratamiento superaron también a los sujetos del grupo de control en todas las mediciones de adaptación personal y social. La evaluación también mostró que el costo del tratamiento psicosocial fue más barato que el tratamiento tradicional.

Palabras clave (enfermedad mental, rehabilitación psicosocial, programa comunitario, costo-efectividad)

Abstract

This article recognizes mental illness as a portentous global health problem with significant consequences for the exercise of human rights and gives an account of the evaluation of a program for people affected by mental illness, that focuses on psycho-social rehabilitation under a community-based perspective, developed in Columbus Ohio, USA. The study used an experimental design and found that the subjects under psychosocial treatment performed better than the control group in key outcomes such as amount of re-hospitalizations, number of hospital days, visits to emergency services mental health, and contacts with the criminal justice system. The subjects of the treatment group also exceeded the control group in all measurements of personal and social adaptation. The evaluation also showed that the psychosocial treatment was less expensive than the traditional one.
\end{abstract}

Key words (mental illness, psycho-social rehabilitation, community based program, cost-effectiveness)

\section{Introducción}

Según la Organización Mundial de la Salud (OMS), más de 450 millones de personas en todo el mundo se han visto afectadas alguna vez por alguna enfermedad mental y por problemas de adaptación. Las personas que padecen estos desórdenes son, con frecuencia, objeto de aislamiento social extremo, muy baja calidad de vida y aumento en morbilidad a causa de la falta de tratamiento de enfermedades físicas tales como diabetes, VIH/Sida y cáncer.

Si bien las enfermedades mentales son comunes a todos los países, los servicios que en ellos se procuran son inadecuados, de modo que la mayor parte de esos desórdenes no se diagnostican de manera correcta, ni se tratan con eficacia. Según la OMS, las naciones de ingresos medios y bajos asignan 
menos de $1 \%$ de sus desembolsos totales en salud a las enfermedades mentales. Es así como se produce una enorme brecha mundial entre las necesidades representadas por aquellos que padecen enfermedades mentales y los sistemas de atención disponibles tanto para abordar esas nocivas condiciones como para fomentar una buena salud mental. El resultado de este grotesco descuido es que las personas con enfermedades mentales siguen soportando el agobio de la estigmatización, los prejuicios y la discriminación. La enfermedad mental, en consecuencia, es un portentoso problema de salud global con importantísimos consecuencias asociadas al ejercicio de derechos humanos. (OMS: http://www.who.int/mental.health/en)

\section{Salud mental}

El Equipo de Evidencias e Investigación (EEI) de la Organización Mundial de la Salud opera a fin de fomentar el mejor entendimiento de las personas con enfermedades mentales y para respaldar el mejoramiento en los sistemas de atención dedicados a ellas El equipo EEI busca además difundir información sobre prácticas basadas en la evidencia para abordar los problemas de salud mental y alentar a las naciones a que adopten políticas que fortalezcan sus sistemas de servicios de salud mental. La OMS piensa que es posible el tratamiento de la enfermedad mental en forma humana y costo-efectiva y que si se aplican métodos probados y eficaces, los individuos afectados estarán en condiciones de adaptarse en forma más eficaz en sus comunidades y de producir importantes mejoras en su calidad de vida.

Con el fin de fomentar un enfoque metodológico acertado del tratamiento de individuos con enfermedades mentales crónicas, la OMS dio inicio al primero de los foros globales sobre servicios de rehabilitación psico-social con base en la comunidad. Considerada una alternativa al hospital psiquiátrico, la rehabilitación psico-social se propone ayudar a las personas afectadas a sobrellevar sus incapacidades en forma más eficaz y a buscar las formas de mejorar su calidad de vida manteniéndose en sus comunidades. Se trata de un enfoque que pone el acento en una filosofía basada en las fortalezas, que alienta a los clientes con enfermedades mentales a ir en pos de metas de recuperación en términos de vivienda, empleo y relaciones sociales. Este enfoque le resta importancia a la enfermedad y a la incapacidad; fomenta las fortalezas de las personas y sus capacidades de desenvolverse con éxito en el entorno de su mundo real.

A fin de obtener un retrato de los programas de re- habilitación psico-social vigentes en el mundo, el foro auspiciado por la OMS solicitó informes provenientes de proveedores de servicios de salud mental en todo el mundo, quienes deberían relatar sus experiencias tanto positivas como negativas en esos programas. Esta solicitud se inició el 31 de Mayo de 2005 y se cerró el 30 de Septiembre de ese mismo año. La OMS tiene planes de publicar algunas de esas contribuciones y de planificar en el futuro una conferencia internacional centrada en la práctica de la rehabilitación psico-social aplicada a personas con enfermedades mentales. Al anunciar este foro la OMS se refiere a la rehabilitación psico-social como el "avance más importante en el servicio a personas con enfermedades mentales graves", y señala que es coherente con el Informe Mundial de la Salud de 2001 que recomienda el reemplazo de los grandes hospitales psiquiátricos por instalaciones de rehabilitación psico-social comunitarias.

Sien el mundo tomarálugar la clausura de los hospitalespsiquiátricos, deberánmejorarsesimultáneamente los servicios psico-sociales con base comunitaria, de otro modo es altamente probable que esas clausuras aumenten el estrés familiar, la carencia de hogar y la extensa enajenación y desesperación personal. (OMS: http://www.who.int/mediacentre/news/releases/2005/pr21/en)

Este artículo informa sobre un muy exitoso programa de rehabilitación psico-social con base comunitaria que se realizó en Columbus Ohio, Estados Unidos. El programa fue el producto de un alto nivel de colaboración y cooperación entre proveedores de servicios de atención de salud mental y la Facultad de Trabajo Social de la Universidad del estado de Ohio. Como se verá en los próximos párrafos, dos aspectos de este proyecto son especialmente dignos de destacarse. En primer lugar, la vasta colaboración entre las agencias que participaron conformó un sistema de servicios integrados, sensibles y costo-eficientes. En segundo lugar, el programa se evaluó rigurosamente mediante un experimento en terreno diseñado expresamente que distribuyó en forma aleatoria las asignaciones tanto de los grupos de tratamiento como de control.

\section{Resumen de la colaboración en el proyecto, fase 1}

La base colaborativa de este proyecto se extendió sobre dos fases. En la Fase 1, el equipo de liderazgo de la Asociación de Salud Mental del Condado de Franklin (Mental Health Association of Franklin County, MMAFC) y la Alianza Nacional pro Enfer- 
mos Mentales del Contado de Franklin (National Alliance for the Mentally Ill of Franklin County, NAMI-FC) buscaron y obtuvieron una subvención federal de la Administración de Servicios de Atención de Salud Mental y Abuso de Sustancias (Substance Abuse and Mental Health Services Administation, SAMHSA) para explorar junto con los participantes de la comunidad de servicios de atención en salud mental locales la posibilidad de crear y poner a prueba un nuevo programa de rehabilitación psico-social en Columbus para personas con enfermedades mentales graves (EMG). El programa propuesto debía modelarse después de Agencia Village de Servicios Integrados (Village Integrated Services Agency, ISA) de Long Beach, CA, reconocida en la bibliografía profesional como "un referente sobre metodología acertada" (Ragins, 2005 y Bibliografía de Village, 2005). La aspiración del proyecto en su Fase I fue desarrollar un alto nivel de consenso entre los líderes de la salud mental de la comunidad local con respecto a la implementación de una réplica de The Village ISA en Columbus, $\mathrm{OH}$., con algunas modificaciones que podrían requerirse en virtud de la diferencia en la base de financiamiento, el sistema real de distribución y el contexto social del entorno.

Durante la Fase I, MHAFC y NAMI-FC coordinaron una serie de reuniones y capacitaciones con veintiún diferentes agencias, organizaciones e individuos con la misión de generar, o interesados en obtener, servicios para enfermos mentales graves en los condados de Franklin y Columbus. El cuerpo docente y los estudiantes de la Facultad de Trabajo Social de la Universidad del Estado de Ohio, dirigidos por el autor de este artículo, ayudaron a facilitar estas reuniones y realizaron evaluaciones y diagnósticos del movimiento del grupo hacia la creación de consenso. Las reuniones con los veintiún actores interesados se sostuvieron sobre una base mensual durante la Fase I. Además de las reuniones mensuales, los actores interesados participaron en un ejercicio de grupos focales para analizar las fortalezas y debilidades en la actual entrega del servicio. Personas con enfermedades mentales, abogados, miembros de sus familias, proveedores de servicios de atención en salud mental de la comunidad, legisladores y el directorio de financiamiento del condado, participaron en los grupos focales de actores interesados. Las conversaciones del grupo focal se centraron en las fortalezas y debilidades de la actual entrega de servicios en salud mental en el Condado de Franklin, así como en la factibilidad de replicar los servicios de recuperación tal como lo hizo The Village, ISA. Se guardaron los registros de las reuniones con propósitos de documentación de los problemas que debían abordarse y para realizar un seguimiento del progreso.

El proceso para lograr consenso emprendido en la Fase I resultó en un estado de buena disposición entre los integrantes con respecto a la implementación de un modelo de servicios integrado en el Condado de Franklin y condujo directamente al desarrollo de una solicitud de subvención ante la Administración de Servicios de Atención de Salud Mental y Abuso de Sustancias (Substance Abuse and Mental Health Services Administration, SAMHSA) en busca de fondos que ayudaran a financiar los costos de la réplica. Si bien la evidencia final de este consenso fue clara y decisiva, el proceso de este proyecto estuvo cargado de considerable tensión y conflictos declarados. Los directores de tres organizaciones expresaron una fuerte oposición a la implementación del proyecto propuesto tanto en el terreno de lo ideológico -The Village ISA no era coherente con las metodologías de tratamiento de las personas con EMG utilizadas en la actualidad- y en el terreno de lo financiero, es decir, el nuevo programa podría restar recursos de los presupuestos actuales. Un total de once reuniones regulares con los actores interesados realizaron entre Diciembre de 2000 y Noviembre de 2001.En el análisis final las mediciones trimestrales de consenso entre los actores interesados - definidas como conocimiento sobre The Village, ISA, factibilidad y respaldo hacia la implementación - demostraron un progreso sustantivo en dirección positiva durante las once reuniones De los 21 actores interesadoss originales, sólo se registró una objeción formal al proyecto propuesto de una réplica piloto.

Animado por este consenso, se formó un subgrupo a partir de los actores interesados originales para formular un plan para la réplica de The Village. Este subgrupo consistió en ocho miembros que representaron a los clientes y miembros de sus familias, abogados de los clientes, especialistas en vivienda y empleo y profesionales de la salud mental. El producto del trabajo de este subgrupo sirvió de base para la solicitud de subsidio al SAMHSA para replicar The Village, la Fase II, y además como un plan para la asociación bajo la cual operaría el proyecto.

El subsidio de SAMSHA para la Fase II fue otorgado en Octubre de 2003, por un importe de 150.000 dólares ${ }^{1}$. El subsidio posibilitó que la colaboración

1 Subsidio \# A0527G1 de los Programas de Subsidios a la Acción Comunitaria de la Administración de Servicios de Salud Mental y Abuso de Substancias. 
prosiguiera con la réplica de The Village como un experimento en terreno rigurosamente evaluado.

\section{La colaboración continúa: Fase 11}

Como se mencionó antes, el programa en discusión se diseñó para reproducir las características sobresalientes de la Agencia de Servicios Integrados The Village de Long Beach, California, en un contexto con base comunitaria en donde a los clientes con enfermedades mentales graves (EMG) se les ofrecería acceso integral a tres diferentes tipos de servicios: integración a la comunidad, servicios de vivienda, y programas vocacionales. Estos esfuerzos coordinados involucraron a cinco organizaciones comunitarias separadas. Dos de estas instituciones, La Asociación de Salud Mental del Condado de Franklin y NAMI - Condado de Franklin, ofrecieron servicios administrativos fiscales y de gestión y trabajaron para asegurar el apego al diseño. Concord Counseling Services (CCS) - una agencia de consulta y salud mental comunitaria - sirvió de emplazamiento para el programa y proporcionó además al director en terreno para el proyecto, especialistas en integración comunitaria y clientes para el proyecto. Los especialistas en vivienda del programa provinieron de la Red de Vivienda Comunitaria (Community Housing Network, CHN) y el especialista en empleo fue facilitado por el Centro de Alternativas Vocacionales (Center of Vocational Alternatives, COVA).

Para recapitular, las similitudes entre The Village, ISA y el programa de Concord Counseling:

- Ambos atienden a personas con Enfermedades Mentales Graves.

- Ambos atienden a poblaciones con altos niveles de desadaptación social.

- Ambos atienden a poblaciones con elevadas tasas de incidencia de carencia de hogar, implicación en casos de justicia penal, abuso de sustancias y desempleo.

- Ambos ofrecen servicios a través de equipos de profesionales que trabajan en colaboración (llamados coordinadores de servicios personales en The Village y gestionadores de casos en Columbus).Ambos conjuntos de equipos cuentan con un profundo compromiso hacia la filosofía de rehabilitación psico-social y de recuperación.
- Ambos enfatizan las relaciones de adulto a adulto entre su personal y los clientes.

- Ambas fomentan entre los clientes el desarrollo de sólidas relaciones entre iguales.

- Ambos se centran en potenciar la búsqueda de calidad de vida por parte de sus clientes en áreas como la vivienda, empleo, finanzas, recreación y participación en la comunidad.

La evaluación del proyecto se concibió como un experimento en terreno controlado (Kerlinger, 1973). Para comenzar, se obtuvieron cincuenta clientes EMG de Concord Counseling Services en forma aleatoria a partir de una lista de todos los clientes EMG del centro. Para calificar para esta lista, cada cliente debía cumplir con uno o más de los siguientes criterios: (1) antecedentes de carencia de hogar; (2) antecedentes de implicación en casos de justicia penal; (3) baja adaptación vocacional. El universo se estratificó de manera de asegurar que los clientes escogidos aleatoriamente representaran verdaderamente un subconjunto de personas con las secuelas habituales de la enfermedad mental crónica.

Luego de obtener consentimiento informado de todos los sujetos, veinticinco clientes fueron asignados al grupo de tratamiento y veinticinco se asignaron al grupo de control mediante un proceso aleatorio. El Cuadro 1 muestra una comparación entre los dos grupos.

El componente investigativo del programa se interesó en abordar diversas preguntas cuya necesidad de exploración adicional se señala en la literatura sobre rehabilitación psico-social (Barton, 1999). Primero, ¿Pueden los servicios de rehabilitación psico-social ofrecerse en forma eficiente y eficaz por medio de la colaboración de las diversas agencias de salud mental comunitarias? Segundo ¿Cómo se comparan los grupos de tratamiento y de control a través del tiempo en términos de los resultados como vivienda, empleo, encuentros con la fuerza pública, re-hospitalizaciones y niveles generales de adaptación psico-social? Tercero, ¿Cuáles, si es que los hay, son los ahorros que pueden producirse con la aplicación de los servicios de rehabilitación psico-social en comparación con los costos de atención de clientes mediante el programa estándar de servicio ambulatorio ofrecido al grupo de control? 
CUADRO 1

CARACTERÍSTICAS DE LAS DOS MUESTRAS

\begin{tabular}{|l|c|c|}
\hline \multicolumn{1}{|c|}{ Demografía } & Grupo de control (n = 25) & Grupo de tratamiento (n = 25) \\
\hline Raza/Etnia & 5 & 4 \\
Afro-Americana & 20 & 21 \\
Euro-Americana & 12 & 13 \\
\hline Género & 13 & 12 \\
\hline Hombres & & \\
Mujeres & $39.3 / 24-54$ & $33.6 / 20-45$ \\
\hline Rango etario Promedio & $42.4 / 28-55$ & $36.9 / 22-52$ \\
Hombres & $40.9 / 24-55$ & $35 / 20-52$ \\
\hline Mujeres & & 12 \\
\hline Diagnostico psiquiátrico & 2 & 1 \\
\hline Esquizofrenia, todos los tipos & 8 & 6 \\
\hline Trastornos delirantes & 5 & 0 \\
\hline Desorden bipolar, todos los tipos & 1 & 25 \\
\hline Depresión mayor, todos los tipos & 25 & $\bar{x}: 77.76$ \\
\hline Trastornos de ansiedad & & Rango : $3-204$ \\
\hline Total & $\bar{x}: 85.80$ & \\
\hline Cantidad de meses recibiendo & Rango 3-216 & \\
\hline servicios de atención en salud mental & & \\
&
\end{tabular}

\section{Descripción de la intervención psico-social}

Según la revisión bibliográfica actualizada de Barton sobre el tema, los servicios de rehabilitación Psico-Social apuntan a aumentar el papel del desempeño de las personas que padecen enfermedades mentales graves y persistentes a través de un rango de intervenciones sociales, educacionales, ocupacionales, conductuales y cognitivas. Por lo general, estos servicios abordan cuatro ámbitos que incluyen capacitación relativa a adquirir habilidades (tanto sociales como vocacionales), apoyo de pares (clubes, centros sociales, redes grupales), servicios vocacionales (empleo asistido y transitorio, capacitación laboral) y desarrollo de recursos de consumo comunitario (redes de apoyo y defensa manejados por los pacientes, agencias de representantes y negocios manejados por los consumidores). El objetivo de los programas de rehabilitación psico-social es reducir el deterioro funcional de personas con enfermedades psiquiátricas importantes. La filosofía subyacente a estos programas es la de fomentar el fortalecimiento, la esperanza, las opciones, la recuperación y las competencias (Barton, 1999).

Los participantes del grupo de tratamiento se dividieron en grupos de intereses según sus preferencias expresadas en términos de comunidad, actividades recreativas y experiencias sociales y culturales. Estos grupos sostuvieron reuniones formales al menos una vez por semana en un local de la comunidad acordada por todos los integrantes de cada grupo. Estos grupos consistían en tres a cuatro participantes y por lo general contaron con la compañía de un miembro del personal del proyecto. Se publicó un calendario de actividades comunitarias con una semana de anticipación, y el personal informó a los usuarios acerca de esas oportunidades y los animó a participar.

Como se señaló, los tipos de salida/actividades grupales que se planificaron se basaron en los intereses de los clientes. Se ofrecieron entre 2 y 5 actividades grupales por semana, incluyendo conciertos musicales, comidas, visitas a museos, caminatas en parques comunitarios, salidas de compras, ejercicios, ir al cine, al bowling, eventos de temporada y fiestas grupales. Aunque la mayor parte de estas actividades se llevaron a cabo en el contexto de grupos pequeños, algunas actividades fueron realizadas por usuarios individuales. Los ejemplos de actividades individuales incluyeron el ser el anfitrión de una fiesta, matricularse en un curso, hacer planes con un amigo/a, ingresar a un gimnasio, conseguir una mascota, tomar un curso de artesanías, etc. 
Los especialistas vocacionales y de vivienda aprovecharon las oportunidades que surgieron en los eventos comunitarios y en las sesiones individuales para hablar con los sujetos acerca de sus preferencias de vivienda y sus intereses vocacionales. Los especialistas en vivienda hicieron tomar conciencia a los sujetos respecto de sus opciones de viviendas asequibles y seguras. En forma similar, los especialistas vocacionales se reunieron en forma regular con los sujetos para analizar sus problemas de empleo, así como los problemas no relacionados con el trabajo que podrían interferir con la búsqueda y preparación para éste.

El grupo de control recibió gestión de casos, consulta siquiátrica, servicios de enfermería, servicios de consulta telefónica 24 horas al día, 7 días a la semana (24/7) y terapia individual y grupal. Las enfermeras proporcionaron la administración de las medicinas. Estos servicios también estuvieron disponibles para los sujetos del grupo de tratamiento, por cierto, pero exceptuando la administración de medicinas, no hicieron uso de estos servicios en un grado considerable.

Las mediciones se realizaron al comienzo del programa (periodo inicial) y en forma trimestral tanto en el grupo de tratamiento $(n=25)$ como en el grupo de control $(n=25)$. Las variables de mediciones de resultados incluyen: re-hospitalizaciones; cantidad de días internado; cantidad de visitas de urgencia por problemas de salud mental; contactos con la fuerza pública, estatus de empleo y vivienda. Además, se empleó un nivel de escala operativa para evaluar las habilidades de cuidado personal de los participantes, sus niveles de socialización, sus actividades relacionadas con el cumplimiento de sus responsabilidades en el hogar, compras, manejo de finanzas personales, uso del transporte público, etc. La satisfacción del usuario con los servicios ofrecidos también se evaluó sobre una base trimestral. Por último se desarrolló una escala general de adaptación a fin de captar el tránsito de los clientes a lo largo de los puntos de medición.

La hipótesis general bajo examen era que los participantes del grupo de tratamiento superarían a los sujetos del grupo de control en todos los resultados y mediciones de adaptación personal descritas antes. Se esperaba además, que estos resultados demostraran que múltiples agencias podían colaborar entre sí con éxito bajo un programa común de rehabilitación psico-social sobre una base asequible en lo financiero.

\section{Resultados del proyecto}

Como lo evidencia el Cuadro 2, el grupo de tratamiento en rehabilitación psico-social superó a los miembros del grupo de control del proyecto en cuanto al total de días de re-hospitalización durante el transcurso del proyecto, cantidad de visitas de

CUADRO 2

RE-HOSPITALIZACIONES, VISITAS DE URGENCIA A LA ATENCIÓN EN SALUD MENTAL, CONTACTOS CON LA JUSTICIA PENAL

\begin{tabular}{|c|c|c|c|c|}
\hline $\begin{array}{l}\text { Total de días de re-hospitalización } \\
\text { Grupo de control }(\mathrm{n}=25) \\
\text { Grupo de tratamiento }(\mathrm{n}=25) \\
\text { Totales } \\
\mathrm{x}^{2}=187.718 \text { d.f. }=1 \mathrm{p}<0,0001 \mathrm{C}=0,542549\end{array}$ & $\begin{array}{c}\text { Periodo inicial } \\
64 \\
152 \\
216\end{array}$ & $\begin{array}{c}(\%) \\
29,6 \% \\
70,4 \% \\
100,0 \%\end{array}$ & $\begin{array}{c}1^{\circ} \text { trim. }-4 \text { trim. } \\
216 \\
18 \\
234\end{array}$ & $\begin{array}{r}(\%) \\
92,3 \% \\
7,7 \% \\
100,0 \%\end{array}$ \\
\hline \multicolumn{5}{|l|}{$\begin{array}{l}\text { Cantidad de visitas de urgencia a } \\
\text { la atención en salud mental }\end{array}$} \\
\hline Grupo de control (n=25) & 16 & $37,2 \%$ & 53 & $89,8 \%$ \\
\hline Grupo de tratamiento $(n=25)$ & 27 & $62,8 \%$ & 6 & $10,2 \%$ \\
\hline $\begin{array}{l}\text { Totales } \\
x^{2}=31.469 \text { d.f. }=1 p<0.0001 C=0.485569\end{array}$ & 43 & $100,0 \%$ & 59 & $100,0 \%$ \\
\hline \multicolumn{5}{|l|}{ Cantidad de contactos con la justicia penal } \\
\hline o grupo de control $(\mathrm{n}=25)$ & 16 & $76,2 \%$ & 17 & $100,0 \%$ \\
\hline Grupo de tratamiento $(\mathrm{n}=25)$ & 5 & $23,8 \%$ & 0 & $0,0 \%$ \\
\hline Totales & 21 & $100,0 \%$ & 17 & $100,0 \%$ \\
\hline
\end{tabular}


urgencia a la atención de salud mental y cantidad de contactos con la justicia penal durante el periodo de vida del proyecto. Estas diferencias se refieren tanto a lo estadístico como a lo substancial. Las diferencias en re-hospitalización y visitas de urgencia a atención en salud mental son especialmente destacables ya que el grupo de tratamiento tenía mayor incidencia en ambas en el periodo inicial (Definido como 90 días antes de la fecha de inicio del proyecto).

El estatus de vivienda en el inicio y en el cuarto trimestre se informa en el Cuadro 3. Ambos grupos se mostraron efectivamente a la par en el periodo inicial en términos de carencia de vivienda ( $C s=8$; Ts $=9$ ). La carencia de vivienda se redujo virtualmente a cero en ambos grupos hacia fines del cuarto trimestre $(C s=1 ; T s=0)$. Más individuos del grupo de tratamiento vivían con su familia en el periodo inicial $(C s=0$; $T s=6)$ y este patrón se mantuvo hacia fines del cuarto trimestre $(C s=4 ; T s=10)$. En términos de vivir en forma independiente, diecisiete individuos del grupo de control fueron descritos de ese modo en el periodo inicial en comparación con nueve individuos del grupo de tratamiento. En esta categoría el grupo de tratamiento ganó en cinco hacia fines del cuarto trimestre y el grupo de controles ganó en dos (es decir, Cs = 19; Ts = 14). La vida en hogares colectivos dio cuenta de uno en cada muestra en el cuarto trimestre y sólo uno en el grupo de tratamiento en el periodo inicial.

En relación al estatus de vivienda, la incidencia de carencia de vivienda se redujo a cero en ambos grupos. De este modo, desde la perspectiva de "resultados finales" no puede afirmarse que la dimensión de vivienda del programa ENHANCE haya tenido mayor efecto sobre la carencia de vivienda que el que tuvieron los servicios regulares de consejería y gestión de casos ofrecidos a los clientes por Concord Counseling Services. Sin embargo, los clientes del grupo de tratamiento mostraron mayor estabilidad en cuanto a vivienda, ya que todos ellos contaban con vivienda hacia fines del primer trimestre, mientras que los clientes pertenecientes al grupo de control no contaban con vivienda hacia fines del cuarto trimestre. Además, la condición de vivienda en el grupo de control tuvo menos estabilidad, con un cambio de vivienda en nueve clientes y dos cambios de vivienda en dos clientes durante el transcurso del proyecto en comparación con ningún cambio de vivienda entre los clientes del grupo de tratamiento.

El estatus de empleo de los sujetos se informa en el Cuadro 4. Hubo mucho movimiento hacia fuera de la categoría de "desempleado" en el grupo de tratamiento entre el periodo inicial y el cuarto trimestre $(\mathrm{B}=14 ; \mathrm{Q} 4=1)$, mientras que no hubo movimiento en esta categoría en el grupo de control. $(\mathrm{B}=18$; $\mathrm{Q} 4$ $=18)$. Este movimiento tuvo importancia estadística $(\mathrm{p}=, 004)$. La mayor parte del movimiento en la parte de los sujetos en tratamiento se dio hacia el "trabajo voluntario", es decir, de uno a ocho. Estos actos de realizar trabajo voluntario son importantes en términos del avance en el largo camino hacia el objetivo fundamental de lograr un empleo remunerado.

Un considerable movimiento desde el desempleo se dio también en la categoría de "preparación para el empleo", en donde ocho sujetos del grupo de tratamiento encontraron ubicación hacia la conclusión del proyecto en comparación con cuatro individuos del grupo de control. En términos de empleo en el mercado competitivo, los sujetos del grupo de tratamiento se mantuvieron sin cambios entre el periodo

CUADRO 3

RESUMEN DEL ESTATUS DE VIVIENDA

\begin{tabular}{|c|c|c|c|c|c|c|c|c|}
\hline & \multicolumn{2}{|c|}{$\begin{array}{c}\text { Carencia } \\
\text { de vivienda }\end{array}$} & \multicolumn{2}{|c|}{$\begin{array}{l}\text { Viviendo con } \\
\text { familiares }\end{array}$} & \multicolumn{2}{|c|}{$\begin{array}{l}\text { Viviendo en forma } \\
\text { independiente }\end{array}$} & \multicolumn{2}{|c|}{$\begin{array}{l}\text { Hogar } \\
\text { grupal }\end{array}$} \\
\hline & $\begin{array}{l}\text { Periodo } \\
\text { inicial }\end{array}$ & $4^{\circ}$ tri. & $\begin{array}{l}\text { Periodo } \\
\text { inicial }\end{array}$ & $4^{\circ}$ tri. & $\begin{array}{l}\text { Periodo } \\
\text { inicial }\end{array}$ & $4^{\circ}$ tri. & $\begin{array}{l}\text { Periodo } \\
\text { inicial }\end{array}$ & $4^{\circ}$ tri. \\
\hline $\begin{array}{l}\text { Grupo de } \\
\text { control }(n=25)\end{array}$ & 8 & 1 & 0 & 4 & 17 & 19 & 0 & 1 \\
\hline \multirow[t]{2}{*}{$\begin{array}{l}\text { Grupo de tratamiento } \\
(\mathrm{n}=25)\end{array}$} & 9 & 0 & 6 & 10 & 9 & 14 & 1 & 1 \\
\hline & \multicolumn{2}{|c|}{$x^{2}=$ NA d.f. $=1$} & \multicolumn{2}{|c|}{$\begin{array}{c}x^{2}=2.143 \text { d.f. }=1 \\
p=0,143(N S)\end{array}$} & \multicolumn{2}{|c|}{$\begin{array}{c}x^{2}=0.371 \text { d.f. }=1 \\
p=0,541(N S)\end{array}$} & \multicolumn{2}{|c|}{$\mathrm{x}^{2}=$ NA d.f. $=1$} \\
\hline
\end{tabular}


inicial (6) y el cuarto trimestre (6), mientras en el grupo de control éstos bajaron de cinco a dos.

En las mediciones de adaptación personal y social, los sujetos del grupo de tratamiento superaron a los sujetos del grupo de control pese al hecho de que en diversas áreas los sujetos del grupo de tratamiento se clasificaron por debajo de los sujetos del grupo de control en el periodo inicial. Por ejemplo, en términos de "Cuidado Personal", los sujetos del grupo de tratamiento obtuvieron un puntaje promedio de 25,25 en el periodo inicial en comparación con 30,88 entre los sujetos del grupo de control, una diferencia a favor de los individuos del grupo de control que es importante en lo estadístico. Hacia el cuarto trimestre, los sujetos del grupo de tratamiento habían ganado más de siete puntos en esta categoría mientras que los individuos del grupo de control habían perdido más de dos puntos ( $\mathrm{p}=$,00038). El mismo patrón general se observó en las clasificaciones para "Socialización" y "Actividades". Es decir, los sujetos del grupo de tratamiento ganaron substancialmente; los individuos del grupo de control perdieron un poco de terreno o se mantuvieron sin cambios en lo medular. Al combinar estas tres áreas de funcionamiento en un puntaje compuesto para representar el "Nivel Total de Funcionamiento" (Cuadro 5), el promedio de individuos del grupo de tratamiento había ganado treinta puntos entre el periodo inicial y el cuarto trimestre y los sujetos del grupo de control habían disminuido siete puntos. La diferencia en el cuarto trimestre tiene importancia estadística a favor del grupo de tratamiento $(\mathrm{p}=, 0005)$.

CUADRO 4

RESUMEN DEL ESTATUS DE EMPLEO

\begin{tabular}{|c|c|c|c|c|c|c|c|c|c|c|}
\hline & \multicolumn{2}{|c|}{ Desempleados } & \multicolumn{2}{|c|}{$\begin{array}{l}\text { Preparación } \\
\text { para empleo }\end{array}$} & \multicolumn{2}{|c|}{$\begin{array}{c}\text { Experiencia laboral } \\
\text { ocasional remunerada }\end{array}$} & \multicolumn{2}{|c|}{$\begin{array}{c}\text { Trabajo } \\
\text { voluntario }\end{array}$} & \multicolumn{2}{|c|}{ Empleado } \\
\hline & $\begin{array}{c}\text { Periodo } \\
\text { inicial }\end{array}$ & $4^{\circ}$ tri. & $\begin{array}{l}\text { Periodo } \\
\text { inicial }\end{array}$ & $4^{\circ}$ tri. & $\begin{array}{l}\text { Periodo } \\
\text { inicial }\end{array}$ & $4^{\circ}$ tri. & $\begin{array}{l}\text { Periodo } \\
\text { inicial }\end{array}$ & $4^{\circ}$ tri. & $\begin{array}{l}\text { Periodo } \\
\text { inicial }\end{array}$ & $4^{\circ}$ tri. \\
\hline $\begin{array}{l}\text { Grupo de } \\
(\mathrm{n}=25) \text { control }\end{array}$ & 18 & 18 & 1 & 4 & 1 & 1 & 0 & 0 & 5 & 2 \\
\hline \multirow[t]{2}{*}{$\begin{array}{l}\text { Grupo de trata- } \\
\text { miento }(n=25)\end{array}$} & 14 & 1 & 2 & 8 & 2 & 2 & 1 & 8 & 6 & 6 \\
\hline & \multicolumn{2}{|c|}{$\begin{array}{l}x^{2}=8.506 \text { d.f. }=1 \\
p=0,004(S)\end{array}$} & \multicolumn{2}{|c|}{$\begin{array}{l}x^{2}<0.0001 \text { d.f. }=1 \\
p=1.000(--)\end{array}$} & \multicolumn{2}{|c|}{$\begin{array}{l}x^{2}<0.0001 \text { d.f. }=1 \\
p=1.000(--)\end{array}$} & \multicolumn{2}{|c|}{$\begin{array}{l}x^{2}=\text { NA d.f. }=1 \\
p=\text { NA }(--)\end{array}$} & \multicolumn{2}{|c|}{$\begin{array}{l}x^{2}=0.833 \text { d.f. }=1 \\
p=0,361 \text { (NS) }\end{array}$} \\
\hline
\end{tabular}

CUADRO 5

PUNTAJE TOTAL DE NIVEL DE FUNCIONAMIENTO

(Puntaje en Cuidado Personal, Socialización y Actividades)

\begin{tabular}{|c|c|c|c|c|c|c|}
\hline & & $\begin{array}{l}\text { Periodo inicial } \\
\text { (BSLN) }\end{array}$ & $\begin{array}{c}\text { Primer } \\
\text { trimestre }\end{array}$ & $\begin{array}{l}\text { Segundo } \\
\text { trimestre }\end{array}$ & $\begin{array}{c}\text { Tercer } \\
\text { trimestre }\end{array}$ & $\begin{array}{l}\text { Cuarto } \\
\text { trimestre }\end{array}$ \\
\hline Control & $\begin{array}{l}\text { Promedio } \\
\text { EntEstándar } \\
\text { Mínimo } \\
\text { Máximo }\end{array}$ & $\begin{array}{c}109.04 \\
10.37 \\
84 \\
123\end{array}$ & $\begin{array}{l}108.72 \\
12.05 \\
77 \\
125\end{array}$ & $\begin{array}{c}106.64 \\
11.89 \\
84 \\
125\end{array}$ & $\begin{array}{c}104.56 \\
14.75 \\
65 \\
124\end{array}$ & $\begin{array}{l}102.64 \\
15.46 \\
70 \\
125\end{array}$ \\
\hline Tratamiento & $\begin{array}{l}\text { Promedio } \\
\text { EntEstándar } \\
\text { Mínimo } \\
\text { Máximo }\end{array}$ & $\begin{array}{l}84.88 \\
11.28 \\
71 \\
120\end{array}$ & $\begin{array}{c}106.24 \\
7.66 \\
86 \\
120\end{array}$ & $\begin{array}{c}108.64 \\
7.53 \\
86 \\
121\end{array}$ & $\begin{array}{c}111.92 \\
7.39 \\
88 \\
123\end{array}$ & $\begin{array}{l}114.60 \\
4.46 \\
104 \\
122\end{array}$ \\
\hline Prueba-t & & 7.8832 & 0.8686 & -0.7104 & -2.2310 & -3.7171 \\
\hline d.f. & & 48 & 48 & 48 & 48 & 48 \\
\hline Valores-P & & 0.0000 & 0.3894 & 0.4809 & 0.0304 & 0.0005 \\
\hline $\begin{array}{l}\text { ¿Importancia } \\
\text { a nivel } .05 ?\end{array}$ & & S & NS & NS & S & S \\
\hline
\end{tabular}


Las mediciones de "Adaptación General" de los clientes durante los cuatro trimestres se informan en la Figura 1. Estas clasificaciones reflejan la medida de aumento o deterioro de los sujetos de trimestre en trimestre en cuanto a diez áreas diferentes en una escala de $-3 a+3$. Los valores informados en la Figura 1 reflejan las clasificaciones promedio de estas diez áreas durante cada trimestre. Todas las diferen- cias tienen importancia estadística ( $p<, 0001)$.

El patrón que se muestra en la Figura I es señal del alto crecimiento positivo en las áreas clasificadas en el sector de los sujetos del grupo de tratamiento y de disminución o "no ganancia" en el sector de individuos del grupo de control. Este hallazgo es coherente con las clasificaciones para "Nivel Total de Funcionamiento" informadas en el Cuadro 5.

FIGURA 1

PUNTAJE EN CRECIMIENTO EN ADAPTACIÓN GENERAL

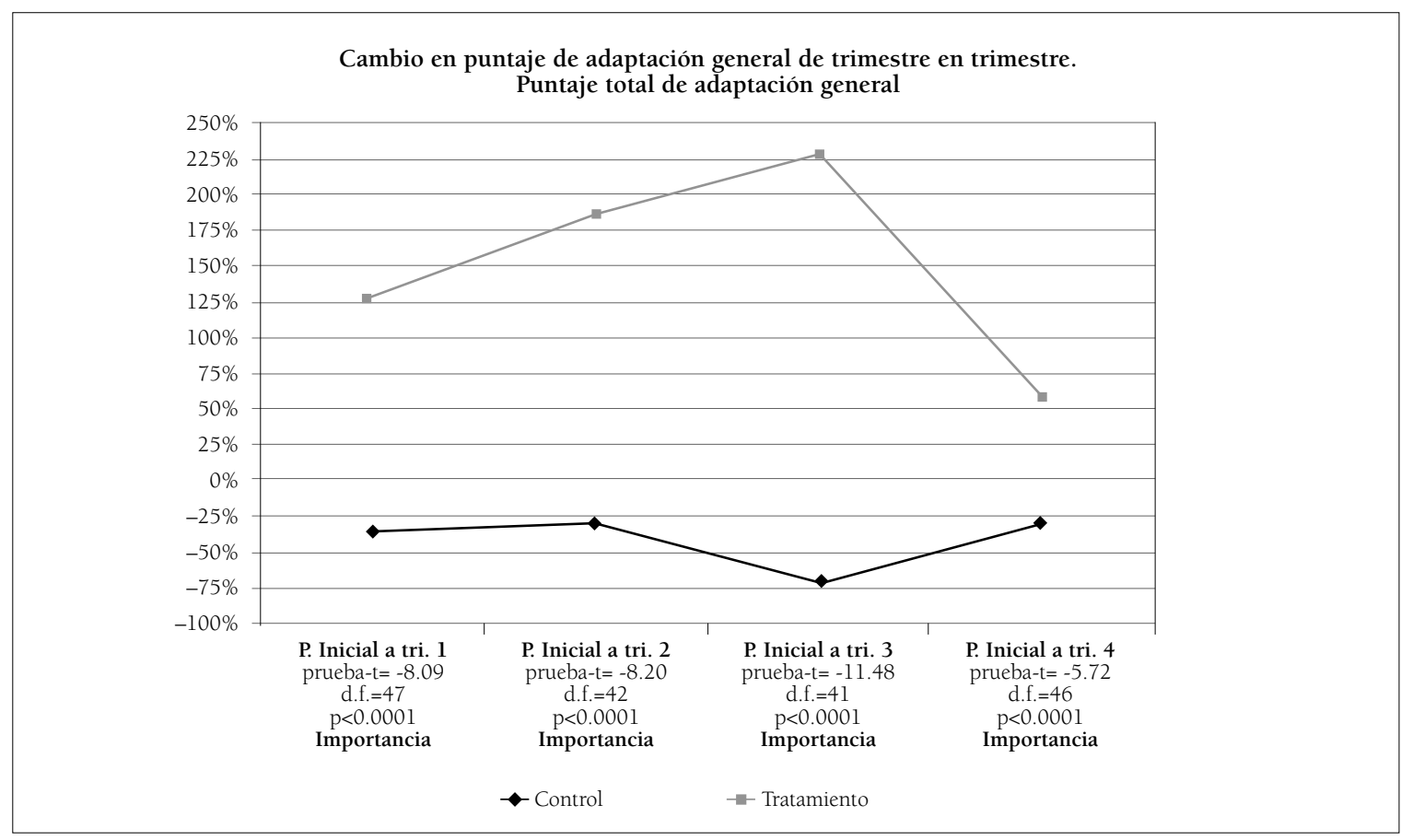

CUADRO 6

PUNTAJE DE SATISFACCIÓN DEL CLIENTE

\begin{tabular}{|c|c|c|c|c|c|c|}
\hline & & $\begin{array}{l}\text { Periodo inicial } \\
\quad(\text { BSLN) }\end{array}$ & Primer tri. & Segundo tri. & Tercer tri. & Cuarto tri. \\
\hline Control & $\begin{array}{l}\text { Promedio } \\
\text { EntEstándar } \\
\text { Mínimo } \\
\text { Máximo }\end{array}$ & $\begin{array}{r}26,40 \\
4,87 \\
15 \\
32\end{array}$ & $\begin{array}{r}25,40 \\
5,56 \\
15 \\
32\end{array}$ & $\begin{array}{r}25,92 \\
5,22 \\
15 \\
32\end{array}$ & $\begin{array}{r}25,88 \\
6,44 \\
14 \\
32\end{array}$ & $\begin{array}{r}24,46 \\
5,44 \\
14 \\
32\end{array}$ \\
\hline Tratamiento & $\begin{array}{l}\text { Promedio } \\
\text { EntEstándar } \\
\text { Mínimo } \\
\text { Máximo }\end{array}$ & $\begin{array}{r}26,52 \\
4,48 \\
16 \\
32\end{array}$ & $\begin{array}{r}25,40 \\
32 \\
4,57 \\
14\end{array}$ & $\begin{array}{r}27,40 \\
3,23 \\
19 \\
32\end{array}$ & $\begin{array}{r}29,32 \\
2,80 \\
21 \\
32\end{array}$ & $\begin{array}{r}29,48 \\
2,40 \\
24 \\
32\end{array}$ \\
\hline Prueba-t & & $-0,0907$ & 0,0000 & $-1,2024$ & $-2,4487$ & $-4,2116$ \\
\hline d.f. & & 48 & 48 & 47 & 48 & 47 \\
\hline Valores-P & & 0,9281 & 1,0000 & 0,2352 & 0,0180 & 0,0001 \\
\hline $\begin{array}{l}\text { ¿Importancia } \\
\text { a nivel } .05 ?\end{array}$ & & NS & NS & NS & $\mathrm{S}$ & S \\
\hline
\end{tabular}


El grado de satisfacción del cliente en el periodo inicial y trimestre a trimestre se informa en el Cuadro 6. El grado de satisfacción comienza con un virtual empate entre los grupos, pero el grupo de tratamiento muestra una mayor satisfacción a lo largo de los trimestres dos, tres y cuatro, mientras que el grupo de control se mantiene sin variaciones. La diferencia en el cuarto trimestre tiene importancia estadísti$\mathrm{ca}(\mathrm{p}<, 0005)$ a favor del grupo en tratamiento (Cs $=24,46 ;$ Ts $=29,48)$.

\section{Conclusiones y análisis}

El proyecto ENHANCE fue una réplica piloto altamente exitosa de The Village, ISA. Los sujetos del grupo de tratamiento superaron a los sujetos del grupo de control en las variables claves de resultados de cantidad de re-hospitalizaciones, cantidad de días de hospitalización, visitas de urgencia a los servicios de salud mental, y contactos con el sistema de justicia penal. Los sujetos del grupo de tratamiento superaron también a los sujetos del grupo de control en todas las mediciones de adaptación personal y social. Se encontró que todas estas diferencias tenían importancia estadística de nivel ,05 o más. Estos hallazgos resultan más notables debido a que en dos de los resultados de las variables -cantidad de días de hospitalización y visitas de urgencia a servicios de salud mental- y en todas las mediciones de adaptación personal, el estatus de los sujetos del grupo de tratamiento era menos favorable que el de los sujetos del grupo de control al momento de las mediciones del periodo inicial.

Hablando en términos prácticos, las hospitalizaciones, visitas de urgencia a los servicios de salud mental y los conflictos con personal de la fuerza pública le sumaron costos a los servicios proporcionados al grupo de control por la comunidad, en los que no se incurrió en relación al grupo de tratamiento. Al calcular los costos diarios e incidentales asociados a estos eventos puede estimarse conservadoramente que durante el transcurso del proyecto, los miembros del grupo de control le costaron al sistema de salud mental entre 102 y 180 mil dólares más que la atención proporcionada a los clientes del grupo de tratamiento. Esta es una diferencia directa en el costo y no incluye los costos indirectos asociados al costo en abogados, tribunales y tiempo de tramitación en oficinas de la fuerzo públicas.

En resumen, este proyecto demostró que era viable la implementación de un modelo de rehabilitación psico-social basado en metodologías acertadas como el de The Village, ISA mediante un esfuerzo conjunto en el que participaran diversas agencias. El proyecto demostró además que una implementación de ese tipo puede lograr resultados superiores en muchos aspectos relativos a la adaptación del usuarioa la comunidad en comparación con el enfoque de tratamiento ambulatorio estándar que por lo general está a disposición de los usuaros con enfermedades mentales graves. Además, esto puede lograrse con importantísimos ahorros en los costos de las agencias que proporcionan los servicios al igual que para la comunidad de salud mental y los sistemas de justicia penal.

\section{Consecuencias internacionales}

El programa de rehabilitación psico-social descrito en este artículo tiene importantes consecuencias en la entrega de servicios eficaces en las poblaciones de enfermos mentales graves de todo el mundo. Los elementos esenciales de este tipo de programas pueden replicarse con un alto grado apego en contextos altamente variados, tanto rurales como urbanos, y tanto en los países desarrollados como en los países en vías de desarrollo. Además, el precio de la implementación de la rehabilitación psico-social es razonable y costo-efectivo.

La implementación exitosa del enfoque de rehabilitación psico-social para ayudar a los enfermos mentales graves depende de la existencia de profesionales bien preparados en servicios sociales, así como de la disponibilidad de profesionales de apoyo en los campos relacionados, tales como el de vivienda y empleo, por ejemplo. Un trabajador social profesionalmente bien preparado debe estar informado tanto de la etiología como de las dinámicas de las enfermedades mentales crónicas así como de los efectos debilitadores de esas enfermedades crónicas. Adicionalmente el trabajador social con la preparación adecuada debe poseer conocimientos y habilidades tanto en los enfoques individuales como grupales de los procesos de ayuda. El foco de estos procesos de ayuda -tanto individual como grupal- debe estar en el refuerzo de las fortalezas personales, el aliento de crecimiento personal y en el desarrollo de sistemas de apoyo mutuo. Finalmente, para funcionar con éxito, un programa de rehabilitación psico-social debe contar con acceso a enfermería psiquiátrica que pueda atender los problemas que surjan así como para administrar los medicamentos.

Dados los recursos, talentos y habilidades profesionales anteriormente descritas, parecería que la ambición de la OMS de fomentar el desarrollo 
mundial de la rehabilitación psico-social efectiva a personas con enfermedades mentales graves es algo que puede materializarse y llevarse a cabo.

\section{Referencias}

BARTON, R. (1999). Psychosocial rehabilitation services in community support systems: A review of outcomes and policy recommendations. Psychiatric Services, 50 (April). 525-534.

KERLINGER, F. N. (1973). Foundations of behavioral research, 2nd ed. NU: Rinehart \& Winston, Inc.
RAGINS, M. (2005). History and overview of The Village. Retrieved from http://www.village- isa.org/ Ragin'\%20Papers/Hist.\%20\&\%20Overview.htm

WORLD HEALTH ORGANIZATION (2005). Mental health and human rights. Retrieved fromhttp://www. who.int/mental_health/en/

WORLD HEALTH ORGANIZATION (2005). The World Health Organization announces first-ever international forum on community mental health services. Retrieved from http://www.who.int/mediacentre/news/releases/2005/ pr21/en/ 